\title{
Arm-Root Curve Fitting Based on Body Surface Feature Points for Young Male
}

\author{
Kaili Liu, Hongshu Jin \\ Wuhan Textile University, Wuhan, Hubei, China \\ Email: 18808695329@163.com,2013025@wtu.edu.cn
}

How to cite this paper: Liu, K.L. and Jin, H.S. (2021) Arm-Root Curve Fitting Based on Body Surface Feature Points for Young Male. World Journal of Engineering and Technology, 9, 241-249. https://doi.org/10.4236/wjet.2021.92017

Received: February 23, 2021

Accepted: April 6, 2021

Published: April 9, 2021

\begin{abstract}
This paper is aiming to obtain an arm-root curve function performing the human arm-root size and shape realistically. A gypsum replica of upper arm for young male was made and scanned for extracting the 3D coordinates of 4 feature points of shoulder point, the anterior/posterior armpit point and the axillary point describing the real arm-root shape under the normalized definitions, and the 5 landmarks were confirmed additionally for improving the fitting precision. Then, the wholly and piecewise fitting of arm-root curve with 9 feature points and mark points in total were generated respectively based on least square polynomial fitting method. Comparing to the wholly fitting, the piecewise fitted function segmented by the line between anterior and posterior axillary points showed a high fitting degree of arm-root morphology with R-square of 1 , the length difference between fitted curve and gypsum curve is $0.003 \mathrm{~cm}$ within error range. And it provided a basic curve model with standard feature points to simulate arm-root morphology realistically by curve fitting for accurate body measurement extraction.
\end{abstract}

\section{Keywords}

Arm-Root Morphology, Curve-Fitting, Feature Point, Piecewise Curve Fitting, Arm-Root Curve Simulation

\section{Introduction}

The body surface feature points are the basic unit for both contact and non-contact measurements for gathering human morphometrical data [1]. Combing with upper garment structure design, the arm-root shape and size are closely related to the armhole structure designing. The feature points pathing through the arm-root curve is not only characterizing the arm-root shape, also is the basis of measuring correctly. 
With rapid growth of online clothing customization, the 3D human body model constructed by point cloud instead of the real human body plays an important role in acquiring the human body sizes [2]. However, as the optical characteristics of the whole-body scanner itself, the scanning blind area of cloud point generally appear in armpit, crotch, and others [3], and the actual body surface characteristics of these areas frequently erased and blurred after processing of repairing, noise reduction and smoothing, and the division between arms and torso is more complicated owing to the diversity of arm-root shape. Therefore, the feature points marked before scanning or measuring manually having the consistency of anthropometric survey and reducing the measurement error.

For improving the accuracy and efficiency of measuring on body surface, Hyunsook Han et al. measured the underarm circumference generated by a b-spline curve using the method of interpolating the front armpit folding point, armpit point, and back armpit folding point on the obscured underarm area, and the armscye circumference was shorter than the manual measuring with large SD [4]. And then, they used the arm and torso intersect point as armpit point which is lower than actual armpit point and the armscye circumference was larger than the manual measuring, especially for an overweight person. Moreover, Danhua Li [5] proposed a feature point extracting method of arm-root according to the point whose gain value is greater than the geometric threshold.

These researches presented the common measurement methods of armscye circumference on processed 3D mannequins currently, although the semantic landmark identifications adopted arm-root curve generations, but it did not reflect the actual curve of underarm due to the surface data unavailable. Besides, the difference in measuring paths and posture in measuring method [6], could be cause the differences in manual measurement on body surface and automatic measurements on mannequins are not fully compatible [7].

Thus, the purpose of this study is to obtain an arm-root curve function performing the human arm-root size and shape realistically. The arm-root's feature points and their definitions is used as expert knowledge for characterizing the arm-root shape. The 3D coordinates of arm-root feature points were extracted from upper arm gypsum replica point cloud are used to obtain arm-root curve by function model fitting method, and it is going to be used as a basic template curve model for various arm-root shape fitting and underarm data renovating.

\section{Research Method}

\subsection{Measurement Subject}

The measurement subject is a 22-year-old male college student, stature of 165 $\mathrm{cm}$ and weight of $60 \mathrm{~kg}$.

\subsection{Definition Feature Points of Arm Root}

In this study, a total of 4 feature points were confirmed to describe the arm-root shape as shown in Figure 1. 


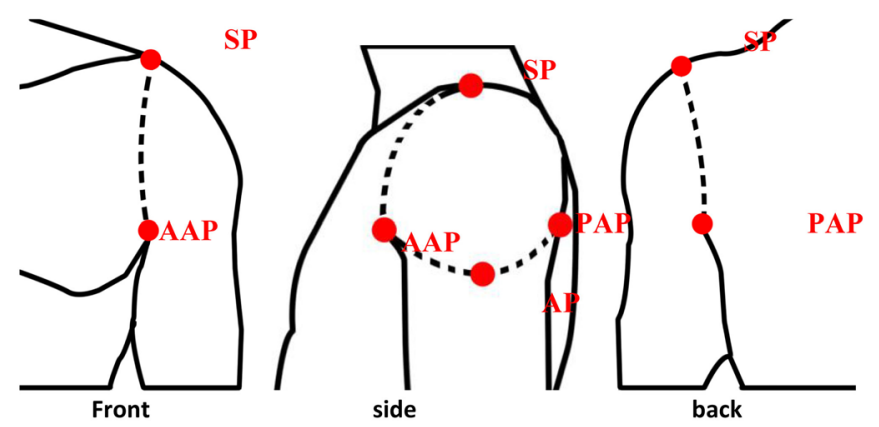

Figure 1. Definitions of arm-root feature points.

Among them, the SP (shoulder point) was defined in accordance with ISO 7250-1 "Basic Human Body Measurements for Technological Design: Body Measurement Definitions and Landmarks" [6]. The AAP (anterior armpit point) and PAP (posterior armpit point) were defined as the anterior upper axillary folding and the posterior upper axillary folding referring to the ergonomic measurement point definition by Machiko MIYOSHI [8]. In addition, the AP (Axillary point) customized the lowest point of the arm-root confirmed from gypsum replica of upper arm in this study.

\subsection{Process of Making Gypsum Replica of Upper Arm}

In order to obtain the arm-root shape completely, the gypsum replica of upper arm was made by the following procedure: 1) Mark the above 4 feature points, arm root curve and upper arm circumference on the upper arm; 2) Cut the plaster bandage into several pieces of $20-30 \mathrm{~cm}$ long, after soaking in warm water, quickly stick it to upper arm surface, then repeat the same three layers; 3) Remove the solidified gypsum replica from upper arm, and remark the feature points and reference lines on inner surface of replica when it dry.

\subsection{Gypsum Replica of Upper Arm Scanning}

The upper arm gypsum replica scanned using GO! SCAN handheld 3D colour scanner for extracting the $3 \mathrm{D}$ point cloud data of upper arm gypsumreplica.

\section{Arm-Root Curve Function Modelling}

\subsection{Extraction of 3D Feature Points Coordinate Data of Arm Root}

The scanned upper arm gypsum replica point cloud data was import into 3DMAX as shown in Figure 2. There are 9 points extracted in total, besides 4 arm root feature points were marked with $0.2 \mathrm{~cm}$ diameter ball, 5 auxiliary points of Z1 - Z5 were added to improve the arm root curve function fitting degree.

The $\mathrm{Z} 1$ is the shoulder lordosis in natural state of arm on the side of torso. Z2 and $\mathrm{Z} 3$ are the intersection points of arm root curve on the horizontal section of the midpoint of vertical distance between SP and AAP. Z4 and Z5 are the intersection points of arm root curve on the horizontal section at $1 / 3$ of the vertical distance between AP and AAP. 


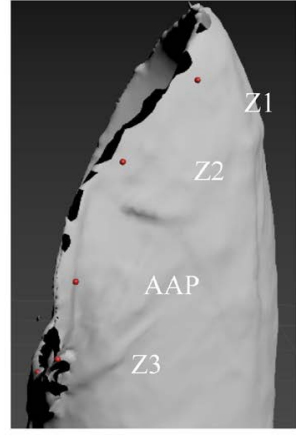

Front of the upper arm

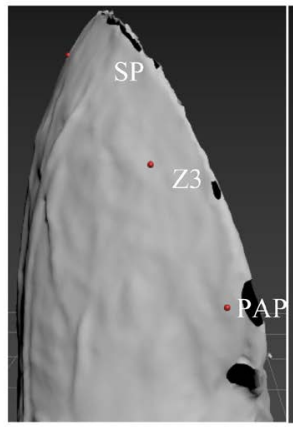

Back of upper arm

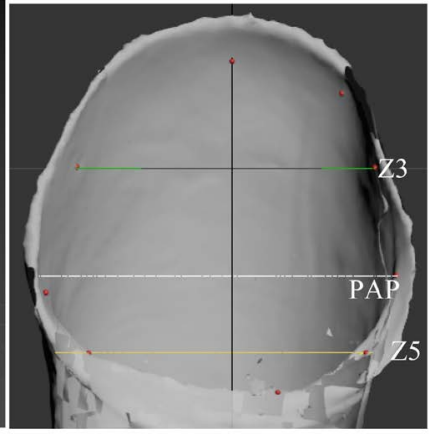

Inside of upper arm

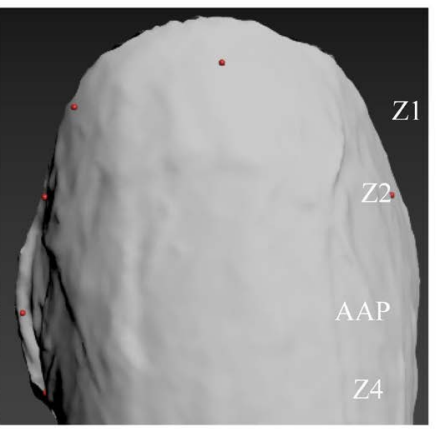

lateral of upper arm

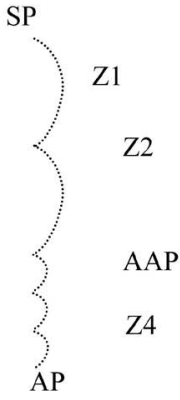

SP

Figure 2. Feature points and auxiliary points marking on arm root.

Then, we imported the feature point, reference lines and arm root curve models into AutoCAD, extracted coordinates of each point using coordinate output commands. The arm-root curve passing through the feature points and auxiliary points generated by B-spline curve tool [9] as shown in Figure 3, which taking the feature point SP as the origin point. After three times feature and mark points 3D coordinate data extraction [10], the average value was $39.2428 \mathrm{~cm}$.

The shoulder point is easily to recognize on body surface [11] convenient for the fitting operation with other feature points, since it located in the prominent position of shoulder.

\subsection{Arm-Root Curve Function Fitting}

In order to obtain the function model of arm-root curve with small error and simulating the arm-root shape realistically [12], the methods of overall curve fitting and segmented curve fitting conducted respectively by the curve fitting tool in MatlabR2018b to comparing the simulation effect using the arm-root feature points and auxiliary points.

\subsubsection{Wholly Arm-Root Curve Fitting}

The arm-root function curve generated based on the mean value of three dimensional coordinates of the feature points and mark points using the least square polynomial fitting method [13], and the fitting results showed in Figure 4.

The 9 points are all distributed on the same micro-convex surface, the function equation is: 


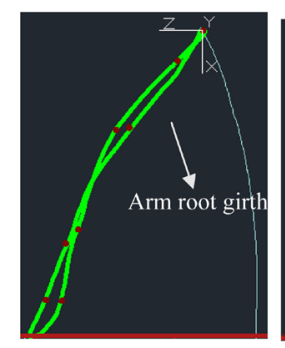

Front of the upper arm

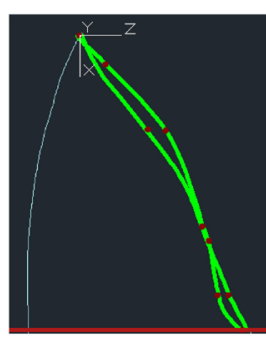

Back of upper arm

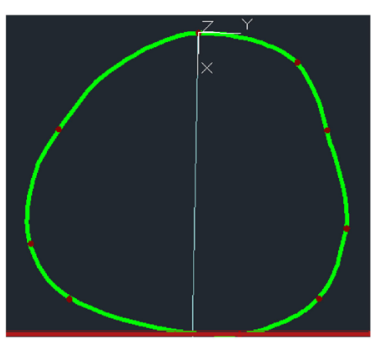

Inside of upper arm

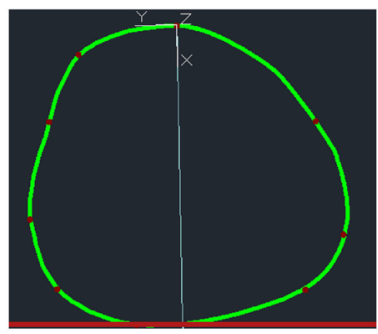

lateral of upper arm

Figure 3. Arm-root B-spline curve extraction with feature point and auxiliary points.

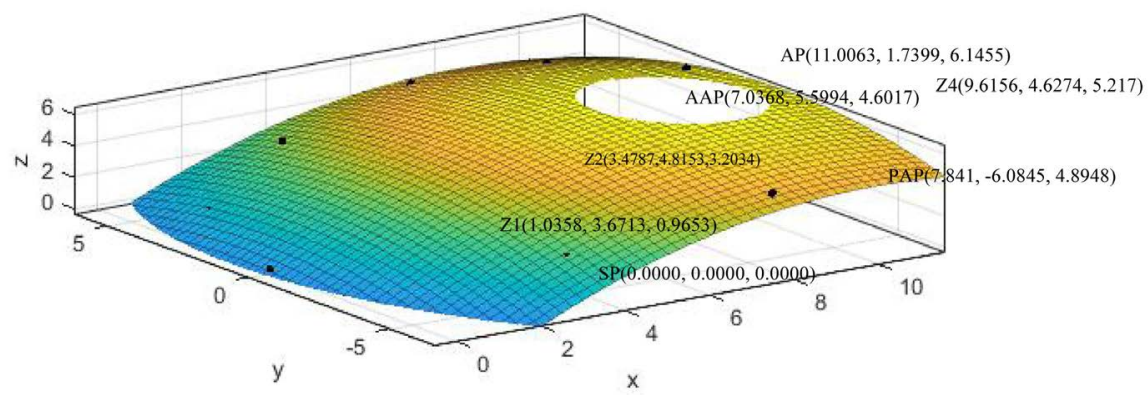

Figure 4. Wholly arm-root curve fitting by polynomial function.

$$
\mathrm{f}(x, y)=\mathrm{p} 00+\mathrm{p} 10 x+\mathrm{p} 01 y+\mathrm{p} 20 x^{2}+\mathrm{p} 11 x y+\mathrm{p} 02 y^{2}
$$

Coefficients (with $95 \%$ confidence bounds):

$$
\begin{aligned}
& \text { p00 }=-0.05414(-0.4198,0.3115) \\
& \text { p10 }=1.469(0.903,2.035) \\
& \text { p01 }=0.1159(0.02591,0.2059) \\
& \text { p20 }=-0.08036(-0.1282,-0.03248) \\
& \text { p11 }=-0.01811(-0.03144,-0.004782) \\
& \text { p02 }=-0.05186(-0.09845,-0.00527)
\end{aligned}
$$

The fitting function equation $f(x, y)$, namely, $\mathrm{Z}$ is the point that is satisfying the function $f(x, y)$ in the coordinates of the XY plane. When $\mathrm{X}$ and $\mathrm{Y}$ were adjusted to second order, the distance between the fitting surface and the points is minimized with fit R-square of 0.9985 .

\subsubsection{Piecewise Arm-Root Curve Fitting}

Generally, the feature points of AAP and PAP were regarded as the demarcation of arm root curve [14], the part above AAP and PAP points is exposed on the body surface, and the part below AAP and PAP points is hidden inside of underarm. Thus, the arm root curve was segmented into two sections of 
AAP-SP-PAP and AAP-AP-PAP for curve function fitting respectively.

\section{1) Under arm-root curve fitting of AAP-AP-PAP}

The underarm curve fitting of AAP-AP-PAP passed through the feature points of AAP, Z4, AP, Z5 and PAP, which distributed on the concave surface, and Figure 5 was showed the curve fitting results using least square polynomial fitting method equally.

The fitted surface of underarm formed concave that is consistent with curve of AAP-AP-PAP, and differs from the monotonic convex shape of the wholly fitting surface as shown in Figure 4.

When $\mathrm{X}$ was adjusted to second order and $\mathrm{Y}$ was adjusted to one order, the distance between the fitting surface and the points is minimized with $\mathrm{R}$-square = 1.000. The function equation is:

$$
f(x, y)=\mathrm{p} 00+\mathrm{p} 10 x+\mathrm{p} 01 y+\mathrm{p} 20 x^{2}+\mathrm{p} 11 x y
$$

Coefficients:

$$
\begin{aligned}
& \text { p00 }=7.739 \\
& \text { p10 }=-0.9784 \\
& \text { p01 }=0.08619 \\
& \text { p20 }=0.07663 \\
& \text { p11 }=-0.01343
\end{aligned}
$$

\section{2) Upper arm-root curve fitting of AAP-SP-PAP}

The upper arm-root curve of AAP-SP-PAP consists of three feature points of AAP, PAP and SP, and three auxiliary points of Z1, Z2 and Z3, and the result was shown in Figure 6. The fitting surface formed a convex surface, and its shape is consistent with the outward of AAP-SP-PAP curve. When X and Y were adjusted to second order, the distance between the fitting surface and the points is minimized with $\mathrm{R}$-square $=1$. The function equation is:

$$
f(x, y)=\mathrm{p} 00+\mathrm{p} 10 x+\mathrm{p} 01 y+\mathrm{p} 20 x^{2}+\mathrm{p} 11 x y+\mathrm{p} 02 y^{2}
$$

\section{Coefficients:}

$$
\begin{aligned}
& \text { p00 }=4.432 \mathrm{e}-15 \\
& \text { p10 }=1.832 \\
& \text { p01 }=0.155 \\
& \text { p20 }=-0.1026 \\
& \text { p11 }=-0.02754 \\
& \text { p02 }=-0.09543
\end{aligned}
$$

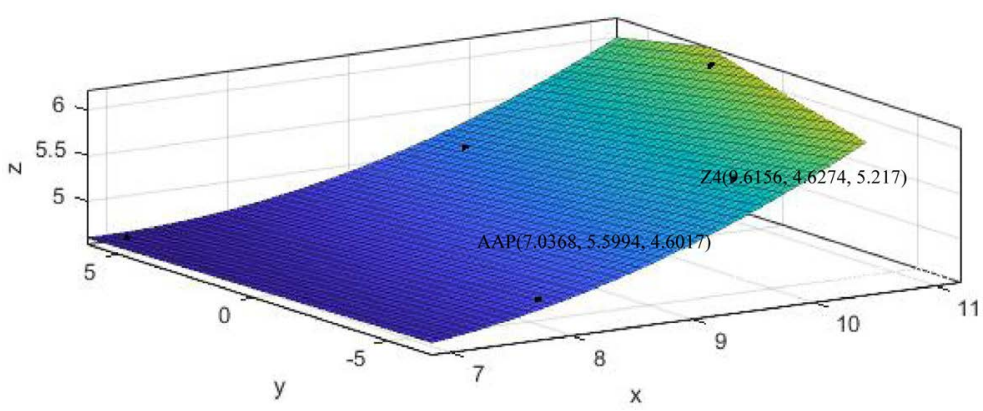

Figure 5. AAP-AP-PAP curve fitting by polynomial function. 


\section{Verification of Arm-Root Curve}

For verifying the approximation of arm-root fitting curves generated by two different method, the fitting curves and replica curve were compared by AutoCAD.

\subsection{Verification of Wholly Arm-Root Fitting Curve}

The wholly arm-root fitting curve and replica curve were overlapped taking the shoulder point as origin point, the overlapped graphic shown in Figure 7. The length of fitted curve is $39.2157 \mathrm{~m}$, which is shorter $0.027 \mathrm{~cm}$ than replica curve. The fitting curve has slightly different in SP-PAP segment from actual arm-root shape.

\subsection{Verification of Piecewise Arm-Root Fitting Curve}

The overlapped graphic of piecewise arm-root fitting curve and replica curve showed in Figure 8 taking SP as the origin point equally. The length of arm root fitting curve is $39.2396 \mathrm{~cm}$, and differed $0.003 \mathrm{~cm}$ from the length of 3D replica curve of $39.2428 \mathrm{~cm}$ within the error range. These two curves had a highly coincidence of replica curve shape, which means that the piecewise fitting function model is effectively in describing the arm-root curve realistically.

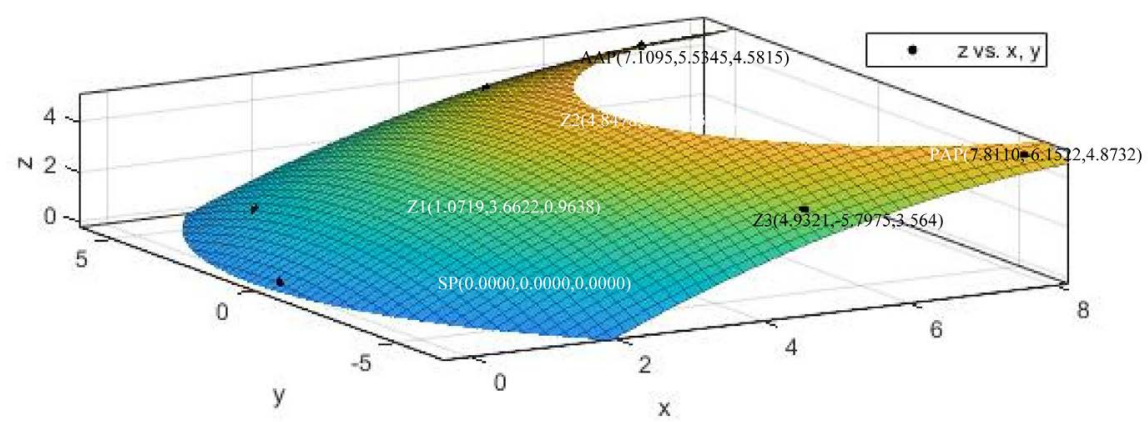

Figure 6. AAP -- SP-- PAP Segment curve fitting by polynomial function.

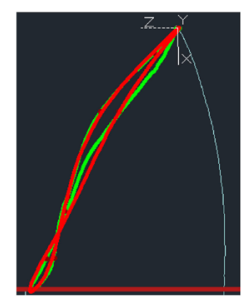

Front of the upper arm

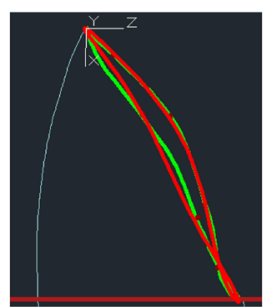

Back of upper arm

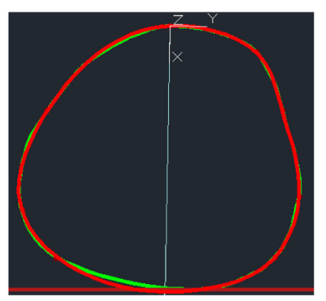

Inside of upper arm

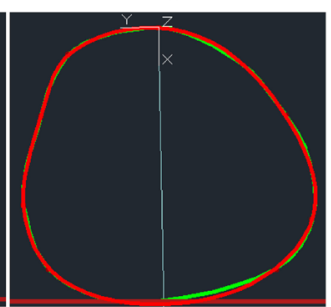

lateral of upper arm gypsum curve

fitting curve

Figure 7. Comparing of wholly fitting curve and replica curve. 


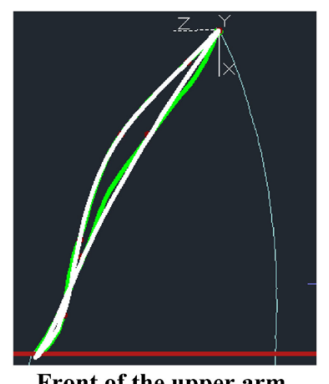

Front of the upper arm

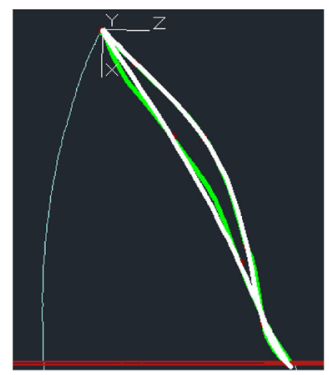

Back of upper arm

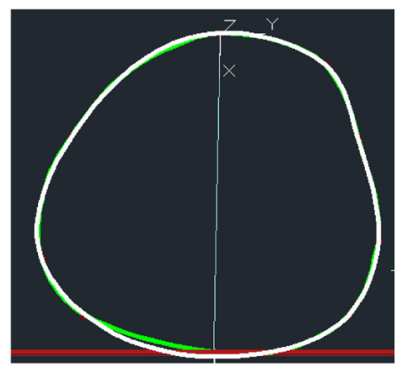

Inside of upper arm

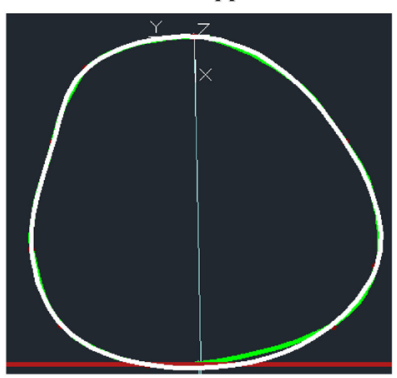

lateral of upper arm

Figure 8. Comparing of piecewise fitting curve and replica curve.

\section{Conclusions and Future Work}

In this study, the wholly and piecewise arm root fitting curve were generated by the three-dimensional coordinates of arm root feature points and auxiliary points from upper arm gypsum replica using the least square polynomial fitting method separately. As the results of comparing, the piecewise arm root curve is closer to the real arm root shape with R-square $=1$, which is proved effectivly.

In the follow-up study, we are going to collect arm roots morphological data based on the surface feature points of arm roots, and establish the morphological feature identification parameters for describing the differences of arm-root shape. Then, we will take the arm-root curve fitting function as the basic model [15] with standard feature points definitions to simulate the individualized arm-root shape by curve fitting method for accurate measurement extraction.

\section{Conflicts of Interest}

The authors declare no conflicts of interest regarding the publication of this paper.

\section{References}

[1] Viviani, C., Arezes, P.M., Bragança, S., et al. (2018) Accuracy, Precision and Reliability in Anthropometric Surveys for Ergonomics Purposes in Adult Working Populations: A Literature Review. International Journal of Industrial Ergonomics, 65, 1-16. https://doi.org/10.1016/j.ergon.2018.01.012

[2] Zhang, X.N., Wang, J. and Zhang, C.Y. (2018) Summary of 3D Human Body Modeling Methods for Digital Clothing. Shandong Textile Science \& Technology, 59, 44-47.

[3] Daanen, H.A.M. and Ter Haar, F.B. (2013) 3D Whole Body Scanners Revisited. 
Displays, 34, 270-275. https://doi.org/10.1016/j.displa.2013.08.011

[4] Han, H. and Nam, Y. (2011) Automatic Body Landmark Identification for Various Body Figures. International Journal of Industrial Ergonomics, 41, 592-606. https://doi.org/10.1016/j.ergon.2011.07.002

[5] Li, D.H. (2016) Research on 3D Human Body Feature Recognition Algorithm Based on Digital Image Processing. Ph.D. Thesis, BIFT (Beijing Institute of Fashion Technology), Beijing.

[6] ISO 7250-1 Basic Human Body Measurements for Technological Design: Body Measurement Definitions and Landmarks.

[7] Markiewicz, L., Witkowsk, M., Sitnik, R. and Mielicka, E. (2017) 3D Anthropometric Algorithms for the Estimation of Measurements Required for Specialized Garment Design. Expert System with Application, 85, 366-385.

https://doi.org/10.1016/j.eswa.2017.04.052

[8] Miyoshi, M. and Milyoshi, M. (2000) The Theory of Fashion Modeling. 1th Edition, China Textile \& Apparel Press, Beijing, 67.

[9] Li, M.Y., Cheng, S.Y., Cong, H.C., Yang, X.R. and Zhang, X.W. (2018) CAD Modeling and Application of Human Body Based on Boundary Division. Modern Manufacturing Engineering, 9, 66-69.

[10] Villamor, E. and Bosch, R.J. (2015) Optimal Treatment of Replicate Measurements in Anthropometric Studies. Annals of Human Biology, 42, 507-510. https://doi.org/10.3109/03014460.2014.969488

[11] Wu, T.F., Wu, X.M., Hu, D., Wang, Q.G. and Wang, F. (2016) Research Progress on Kinematics Analysis of Shoulder Joint Based on Optical Motion Capture System. Chinese Electronic Journal of Shoulder and Elbow Surgery, 4, 248-250.

[12] Ai, R.Y., Zhuang, M.L., Zhang, M.M., Wang, M.M. and Liu, J. (2020) Research on Human Body Surface Modeling Based on Graphics and Images. Knitting Industry, 6, 72-76.

[13] Chen, L.F., Yang, J.Y., Cui, S., Pan, Q.C. and Li, L. (2014) The Least Squares Curve Fitting Simulation Research Based on MATLAB. Journal of Shenyang Normal University (Natural Science), 32, 75-79.

[14] Yoon, M.K., Nam, Y.J. and Kim, W. (2016) Classifying Male Upper Lateral Somatotypes Using Space Vectors. International Journal of Clothing Science and Technology, 28, 115-129. https://doi.org/10.1108/IJCST-03-2015-0043

[15] Zhou, Z.K. and Hao, S.J. (2016) Anatomical Landmark Detection on 3D Human Shapes by Hierarchically Utilizing Multiple Shape Features. Neuro-Computing, 253. https://doi.org/10.1016/j.neucom.2016.09.131 